\title{
Modeling of Salt Tectonics
}

\author{
P. Massimi ${ }^{\text {a }}$ A. Quarteroni ${ }^{\text {a,b }}$ F. Saleri ${ }^{\text {a }}$ G. Scrofani ${ }^{a}$ \\ ${ }^{a} M O X$ - Modeling and Scientific Computing, \\ Dipartimento di Matematica "F. Brioschi" \\ Politecnico di Milano, via Bonardi 9, 20133 Milano, Italy \\ b CMCS (Chair of Modeling and and Scientific Computing) - IACS \\ EPFL ,Station 8, CH-1015 Lausanne, Switzerland
}

Citation information: P. Massimi, A. Quarteroni, F. Saleri, G. Scrofani, Modeling of salt tectonics, Comp. Meth. Appl. Mech. Engrng., Vol 197/1-4

(2007), pp 281-293.

DOI information: 10.1016/j.cma.2007.08.004

\begin{abstract}
In this work a general framework for the simulation of sedimentary basins in presence of salt structures is addressed. Sediments and evaporites are modeled as nonNewtonian fluids and the thermal effects induced by the presence of salt are taken into account. The computational strategy is based on a Lagrangian methodology with intensive grid adaptivity, together with a kinematic modeling of faults and different kinds of boundary conditions representing sedimentation, erosion, basement evolution, lithospheric compression and extension. The proposed methodology is applied to simple test cases as well as to a geological reconstruction of industrial interest.
\end{abstract}

Key words: Salt tectonics, diapirism, non-Newtonian fluids, finite elements, adaptive modeling, multi-physics.

\section{Introduction}

In the past few years salt tectonics has become increasingly important in petroleum exploration industry because of the role it plays in hydrocarbon

Email addresses: paolo.massimi@polimi.it (P. Massimi), alfio.quarteroni@epfl.ch (A. Quarteroni), giovanni.scrofani@polimi.it (G. Scrofani). 
generation and accumulation [54]. In fact the high mobility and low permeability of salt can promote trap and seal formation and the temperature anomalies induced by its high thermal conductivity $[21,22,28]$ can retard maturation of subsalt source rocks and accelerates that of supra salt rocks $[36,35,38,48]$. Some typical examples of the strong interplay between salt tectonics and hydrocarbon reservoirs can be found in the whole area of the Gulf of Mexico, in the Niger Delta and Indonesia [19]. The low permeability and high thermal conductivity of rocksalt can be exploited also for environmental applications (such as radioactive waste disposal and energy production by geothermal source). Seismic profiles and drillings demonstrate that salt diapirs have a wide variety of shapes $[25,20]$, reflecting the different ways in which diapirs interact with the overburden during their growth $[24,53,50]$.

Traditionally, salt diapirism has been studied using Rayleigh-Taylor type models, describing the gravitational instability of fluid layers [12]. These models have been extended to include several factors and relevant mechanisms, such as the effects of topography [41], erosion and sedimentation [43,42,18], and tectonic extension and compression $[13,37,20]$. Initially salt and overburden were numerically modeled like Newtonian layered fluids with high density and viscosity. The comparison between these works and the complex rheological behaviour observed in many laboratory experiments [49,51,52] showed that the Newtonian model was inadequate to describe rock behaviour $[23,56]$.

Some recent studies have incorporated non-linear models in numerical simulations, showing a better fit with rock behaviour $[15,17,40]$.

A key factor in this kind of simulations is the choice of the general framework essentially between Eulerian, Lagrangian and Arbitrary Lagrangian Eulerian (ALE). The Eulerian methodology is based on a fixed grid on the whole domain, so a critical aspect arises when free surfaces and interfaces have to be included. For some class of problems a local grid refinement is commonly used to reduce numerical errors. Different choices can be adopted within this framework for the tracking of interfaces and free surfaces, the most widely used being particle in cell, volume-of-fluids and level set methods. Particular care must be taken when using these tracking methods in order to improve the accuracy and resolution of the geometry. The Lagrangian approach incorporates the interfaces and geometric description by tracking the reference configuration at each step. In this way the material properties are automatically advected with the material particles. This feature simplifies the description of complex materials with respect to the Eulerian case. The main disadvantage of the Lagrangian approach is that repeated application of mesh movements may lead to bad quality or even invalid mesh, thus a partial or total remeshing is required at some time steps. Finally the ALE method is also used for problems with free surfaces and interfaces. However, depending on the formulation, the ALE method inherits features of both Eulerian and Lagrangian methodologies reducing but not completely overcoming the need for remeshing. The Eulerian and ALE approaches have been extensively adopted in the cited works. Recently new numerical techniques with Lagrangian approximation of model 
equations and grid adaptivity have been introduced $[33,40]$.

The computational approach described in this work is based on the Lagrangian method, which is complemented by robust and efficient adaptive meshing techniques. This analysis will be performed in the framework of generalized Newtonian fluids. For a detailed description of the physical setting and applicability to sedimentary basin we refer to [33]. Also the problem of modeling the thermal anomalies induced by the high thermal conductivity of salt diapirs and its interaction with rheologic properties will be addressed.

\section{Continuum formulation for generalized Newtonian fluids}

We focus our attention on non-Newtonian models for the mechanic of sedimentary basins $[8,42,41,18]$. A non-Newtonian fluid is characterized by a non-linear dependence between shear stress and shear rate. If we assume that the Cauchy stress tensor $\boldsymbol{\sigma}$ is frame independent and is a function of density $\rho$ and on the symmetric gradient $\mathbf{D}(\mathbf{u})$ of the velocity $\mathbf{u}$, here indicated simply by $\mathbf{D}$, and that the stress is frame independent, then, thanks to the CayleyHamilton theorem, $\boldsymbol{\sigma}$ has the representation $\boldsymbol{\sigma}=\alpha_{0} \mathbf{I}+\alpha_{1} \mathbf{D}+\alpha_{2} \mathbf{D}^{2}$, where $\alpha_{i}=\alpha_{i}\left(\rho, \mathrm{I}_{D}, \Pi_{D}, \Pi_{D}\right) i=0,1,2$, and

$$
\mathbf{D}=\frac{1}{2}\left(\nabla \mathbf{u}+\nabla \mathbf{u}^{T}\right), \mathrm{I}_{D}=\operatorname{tr} \mathbf{D}, \Pi_{D}=\frac{1}{2}\left[(\operatorname{tr} \mathbf{D})^{2}-\operatorname{tr}\left(\mathbf{D}^{2}\right)\right], \Pi_{D}=\operatorname{det} \mathbf{D} .
$$

Hence, for an incompressible fluid $\mathrm{I}_{D}=0$ and

$$
|\mathbf{D}|^{2}=\sum_{i, j=1}^{3}\left[D_{i j}\right]^{2}=-2 \Pi_{D},
$$

where $|\cdot|$ denotes the Frobenius matrix norm. If we assume that the fluid is incompressible and that stress depends only on the velocity gradient, the Cauchy stress takes the form:

$$
\boldsymbol{\sigma}=-p \mathbf{I}+\hat{\alpha}_{1}\left(\Pi_{D}, \Pi_{D}\right) \mathbf{D}+\hat{\alpha}_{2}\left(\Pi_{D}, \Pi_{D}\right) \mathbf{D}^{2}
$$

where $p$ is a Lagrange multiplier that is introduced to enforce the constraint of the incompressibility.

In this work we consider a particular subclass of fluids derived from (1) which is widely used for geological application and for which the Cauchy stress is given by

$$
\boldsymbol{\sigma}=-p \mathbf{I}+\mu\left(|\mathbf{D}|^{2}\right) \mathbf{D}
$$

where $\mu\left(|\mathbf{D}|^{2}\right)$ can be interpreted as the apparent viscosity, which depends on the second invariant of the rate of deformation tensor $\Pi_{D}$. Then, depending on the chosen viscosity function, the following prototypical models [39] have been proposed: 
a) the power-law model is based on the assumption that the relationship between shear stress and shear rate (on log-log scale) can be often approximated by a straight line over a limited range of shear rate. For this part of the flow curve the apparent viscosity of the fluid is thus given by

$$
\mu(z)=\mu_{0} z^{(r-2) / 2}
$$

where $\mu_{0} \geq 0$ and $r \in(1, \infty)$ are two empirical curve-fitting parameters (the fluid consistency coefficient and the flow behaviour index, respectively). If $r=2$ the power-law model predicts the Newtonian behaviour;

b) the Carreau viscosity model [10] in which

$$
\mu(z)=\mu_{\infty}+\left(\mu_{0}-\mu_{\infty}\right)(1+\lambda z)^{(r-2) / 2}
$$

where $\lambda \geq 0$ and $\mu_{\infty}>0$ are additional curve-fitting parameters. This model predicts Newtonian fluid behaviour if $\lambda=0$ or $r=2$. If we take $r \in(1,2)$ we have pseudo-plastic fluid, instead if $r \in(2, \infty)$ we predict the behaviour of a dilatant fluid.

\subsection{Generalized Stokes equations}

The rise of diapirs in sedimentary basins is mainly caused by a balance between buoyancy and external driving forces and involves time scales of the order of millions of years. On such a time scale rocks and salt can be modeled as highly viscous fluids. Then the description of the evolution of the basin can be cast into the framework of the Rayleigh-Taylor theory describing the evolution of gravitational instability between layered fluids. Let us state the generalized Stokes problem following the introduction of Baranger [4] and Barret $[5,6,7]$ for non-linear Stokes equations. Let $\Omega$ be a bounded and connected open set in $\mathbb{R}^{2}$ (that represents a vertical section of the basin) with a Lipschitz boundary $\Gamma$. We consider the following boundary-value problem:

$$
\begin{aligned}
-\operatorname{div}\left(\mu\left(|\mathbf{D}|^{2}\right) \mathbf{D}\right)+\nabla p=\mathbf{f} & \text { in } \Omega \\
\operatorname{div} \mathbf{u}=0 & \text { in } \Omega
\end{aligned}
$$

completed by suitable boundary conditions (see sect. 2 ), where $\mathbf{f} \in \mathbf{L}^{2}(\Omega)$ is the applied body force, $\mathbf{u}$ the velocity, $p$ the pressure, $\mu=\mu\left(|\mathbf{D}|^{2}\right) \in C^{0}(0, \infty)$ the viscosity of the fluid. Model (4) represents the steady isothermal flow of an incompressible stratified fluid. In the next section we review the fundamental properties of Carreau and power law models. Assuming uniform rheological properties within each layer we can reduce the time-dependence of the evolution exclusively to the dynamics of the interfaces between layers. We will assume the continuity of the velocity field and of the normal stress on the 


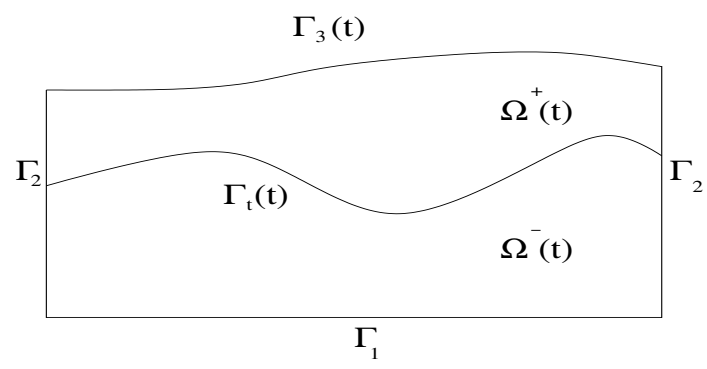

Figure 1. Computational domain

interface $\Gamma_{t}$, separating salt and overburden (see Figure 1). An initial configuration $\Gamma_{0}$ is given for the interface. The evolution of each point belonging to the interface is defined as the solution of the kinematic equation

$$
\left\{\begin{array}{l}
\frac{d \boldsymbol{\xi}}{d t}=\mathbf{u}(\boldsymbol{\xi}), \quad \boldsymbol{\xi} \in \Gamma_{t}, \text { for all } t>0 \\
\boldsymbol{\xi}(0)=\boldsymbol{\xi}_{0}
\end{array}\right.
$$

A physical description of the instability of stratified inhomogeneous fluids (Rayleigh-Taylor instability) can be found in [12]. As a particular case we analyze the evolution of immiscible fluids with different rheological properties (density, viscosity).

\section{Mathematical formulation for the non-linear Stokes problem}

Following [33], we introduce the mathematical formulation for the sedimentary basin evolution, focusing our attention on the non-linearity of the stress tensor. We describe the basin evolution using a non-linear Stokes system in $\Omega(t) \subset \mathbb{R}^{2}$ with moving interface $\Gamma_{t}$ for $t \in(0, T]$, referring to Figure 1 for simplicity. In the sequel the time dependence of the domain and of its boundaries is understood. Equations (5) and (4)will be cast in the general frame of a fluid with variable, possibly discontinuous, density and viscosity. In this context the interface $\Gamma_{t}$ separating $\Omega^{+}$and $\Omega^{-}$is characterized as the loci of discontinuity

of the density $\rho$. The evolution model for basin structures can be formulated 
as follows, for any $t \in(0, T]$ :

$$
\begin{array}{ll}
-\operatorname{div} \mathbf{T}\left(\mathbf{u}, \mu\left(|\mathbf{D}|^{2}\right)\right)+\nabla p=\mathbf{f} & \text { in } \Omega, \\
\operatorname{div} \mathbf{u}=0 & \text { in } \Omega, \\
\frac{\partial \rho}{\partial t}+\mathbf{u} \cdot \nabla \rho=0 & \text { in } \Omega, \\
\mathbf{u}=\mathbf{0} & \text { on } \Gamma_{1}, \\
\mathbf{u} \cdot \mathbf{n}_{2}=0, \quad(\mathbf{T}-p \mathbf{I}) \mathbf{n}_{2} \cdot \mathbf{t}_{2}=0 & \text { on } \Gamma_{2}, \\
(\mathbf{T}-p \mathbf{I}) \mathbf{n}_{3}=-p_{e} \mathbf{n}_{3} & \text { on } \Gamma_{3}, \\
\left.\rho\right|_{t=0}=\rho_{0} & \text { in } \Omega_{0},
\end{array}
$$

where $\Omega=\overline{\Omega^{+} \cup \Omega^{-}}, \partial \Omega=\Gamma_{1} \cup \Gamma_{2} \cup \Gamma_{3}, \Omega_{0}=\Omega(0), \mathbf{T}\left(\mathbf{u}, \mu\left(|\mathbf{D}|^{2}\right)\right)=\mu\left(|\mathbf{D}|^{2}\right) \mathbf{D}$ is the non-Newtonian stress tensor and $\mu\left(|\mathbf{D}|^{2}\right)$ will be a constant if the fluid is Newtonian, $\mathbf{I}$ is the identity tensor, $\mathbf{f}=\rho \mathbf{g}, p_{e}: \Omega \rightarrow \mathbb{R}$ (external pressure) is a given function, $\mathbf{n}_{i}$ and $\mathbf{t}_{i}$ are respectively the unit outward normal and tangent to $\Gamma_{i}$. Free surface boundary condition is prescribed on $\Gamma_{3}$.

In order to model the basin evolution focusing on salt structures, we are interested in the particular case in which the initial function $\rho_{0}(\mathbf{x})$ is positive and discontinuous along $\Gamma_{t}$ (see [34,33]). Considering the fluids immiscible, the problem of advection of density is equivalent to finding the evolution of the moving boundary $\Gamma_{t}$. At the initial time $t=0$, the density is piecewise constant and assumes two positive values characterizing the distinct phases of the flow,

$$
\rho(\mathbf{x}, 0)=\rho_{0}(\mathbf{x})=\left\{\begin{array}{l}
\rho_{+}, \mathbf{x} \in \Omega_{0}^{+} \\
\rho_{-}, \mathbf{x} \in \Omega_{0}^{-}
\end{array}, \rho_{+}>\rho_{-}>0 .\right.
$$

In this case, the condition for density is equivalent to specifying the interface $\Gamma_{t}$ that separates the two subdomains $\Omega_{0}^{+}$and $\Omega_{0}^{-}$initially occupied by different fluids. For the solution of problem (6) we anticipate that we will use the following splitting algorithm: at every time-step $t^{n}$, first we compute the velocity field solving the Stokes problem, then we track the interface $\Gamma_{t}$ to update its position:

$$
\begin{aligned}
& \Gamma_{t}^{n} \longrightarrow\left[\Omega^{+}, \Omega^{-}\right]^{n} \longrightarrow \rho_{+}^{n}, \rho_{-}^{n} \longrightarrow \overline{\Omega^{n}}=\overline{\left[\Omega^{+}\right]^{n}} \cup \overline{\left[\Omega^{-}\right]^{n}} \\
& \left.\longrightarrow \begin{array}{c}
\text { Stokes }\left(\Omega^{n}\right) \longrightarrow\left(\mathbf{u}^{n}\right) \\
\text { (non-linear subiterations) }
\end{array}\right]\left\{\operatorname{Tracking}\left(\Gamma_{t}^{n}, \mathbf{u}^{n}\right) \longrightarrow \Gamma_{t}^{n+1}\right\}
\end{aligned}
$$

where by $\operatorname{Stokes}\left(\Omega^{n}\right)$ we mean that we look for the weak solution of the nonlinear problem on the whole domain (see the next section) and by tracking $\left(\Gamma_{t}^{n}, \mathbf{u}^{n}\right)$ 
we mean that we look for the solution of problem (5).

\subsection{Well posedness results of the generalized Stokes problem}

We adopt the standard notation $W^{r, s}(\Omega)$ for the Sobolev spaces with $r$-th order of differentiation and $s$-th order of integration on the regular domain $\Omega \subset \mathbb{R}^{2}$, with the usual norms $\|\cdot\|_{W^{r, s}(\Omega)}$ and semi-norms $|\cdot|_{W^{r, s}(\Omega)}$. When used for vector-valued functions, these spaces and norms are indicated $\mathbf{W}^{r, s}(\Omega)$, $\|\cdot\|_{\mathbf{W}^{r, s}(\Omega)}$ and $|\cdot|_{\mathbf{W}^{r, s}(\Omega)}$, respectively [1]. We set, for $s \geq 2$,

$W_{0}^{1, s}(\Omega)=\left\{w \in W^{1, s}(\Omega): w=0\right.$ on $\left.\Gamma\right\}, L_{0}^{s}(\Omega)=\left\{w \in L^{s}(\Omega): \int_{\Omega} w d \Omega=0\right\}$.

To introduce the direct and the mixed formulation for the generalized Stokes system $[4,46]$, let

$$
J(\mathbf{v}) \equiv J_{r}(\mathbf{v})-(\mathbf{f}, \mathbf{v}), \text { with } J_{r}(\mathbf{v})=2 \int_{\Omega} \int_{0}^{|\mathbf{D}(\mathbf{v})|^{2}} \mu(z) d z d \Omega,
$$

where $\mu(z)$ is given by (2) or (3), the subscript $r$ refers to power-law or Carreau model exponent. In particular, for the power law (2) we obtain

$$
J_{r}(\mathbf{v})=\frac{4 \mu_{0}}{r} \int_{\Omega}|\mathbf{D}(\mathbf{v})|^{r} d \Omega
$$

while for the Carreau law (3) we have

$$
J_{r}(\mathbf{v})=2 \mu_{\infty} \int_{\Omega}|\mathbf{D}(\mathbf{v})|^{2} d \Omega+\frac{2}{\lambda r}\left(\mu_{0}-\mu_{\infty}\right) \int_{\Omega}\left(\left(1+\lambda|\mathbf{D}(\mathbf{v})|^{2}\right)^{r / 2}-1\right) d \Omega .
$$

Noting that, for a suitable $C(s, m)>0$,

$$
\left|\sum_{i=1}^{m} a_{i}\right|^{s} \leq C(s, m) \sum_{i=1}^{m}\left|a_{i}\right|^{s} \quad \text { for all } s>0 \quad \text { and } \quad m \in \mathbb{N}
$$

for every sequence of real numbers $\left\{a_{i}\right\}$, we can deduce that the functional $J$, is well defined on $\mathbf{W}^{1, s}(\Omega)$ where

$$
\begin{aligned}
& s=2 \text { if } \mu \text { is given by (3) and } r \in(1,2) \\
& s=r \text { if } \mu \text { is given by (2) or if } \mu \text { is given by (3) and } r \geq 2 .
\end{aligned}
$$

For the sake of simplicity we set $\mathbf{X}=\mathbf{W}_{0}^{1, s}(\Omega)$ equipped with the norm $\|\cdot\|_{\mathbf{X}}=$ $|\cdot|_{\mathbf{W}_{0}^{1, s}(\Omega)}$, where $s$ is given in $(7)$. Let $\langle\cdot, \cdot\rangle$ denote the duality paring between 
$\mathbf{X}^{*}$ and $\mathbf{X}$. Then $J$ is Gateaux differentiable on $\mathbf{X}$ with (see [4])

$$
\left\langle J^{\prime}(\mathbf{w}), \mathbf{v}\right\rangle=a(\mathbf{w} ; \mathbf{w}, \mathbf{v})-(\mathbf{f}, \mathbf{v}) \quad \forall \mathbf{w}, \mathbf{v} \in \mathbf{X}
$$

where, for every $\mathbf{u}, a(\mathbf{u} ; \cdot, \cdot)$ is the following continuous bilinear form

$$
a(\mathbf{u} ; \mathbf{v}, \mathbf{w})=2 \sum_{i, j=1}^{2} \int_{\Omega} \mu\left(|\mathbf{D}(\mathbf{u})|^{2}\right) D_{i j}(\mathbf{v}) D_{i j}(\mathbf{w}) d \Omega .
$$

Moreover $J^{\prime}$ is strictly monotone, thus $J$ is strictly convex on $\mathbf{X}$ and $J$ is coercive on $\mathbf{X}$. Setting

$$
\mathbf{V}=\{\mathbf{v} \in \mathbf{X}: \operatorname{div} \mathbf{v}=0 \text { in } \Omega\}
$$

it follows that there exists a unique solution of the minimization problem:

$$
\text { find } \mathbf{u} \in \mathbf{V}: \quad J(\mathbf{u}) \leq J(\mathbf{v}) \quad \forall \mathbf{v} \in \mathbf{V}
$$

Furthermore, problem (8) is equivalent to the Euler equation:

$$
\text { find } \mathbf{u} \in \mathbf{V}: \quad a(\mathbf{u} ; \mathbf{u}, \mathbf{v})=(\mathbf{f}, \mathbf{v}) \quad \forall \mathbf{v} \in \mathbf{V}
$$

One can regard problem (9) as a direct weak formulation of (4). If we set $M=L_{0}^{s^{\prime}}(\Omega)$, with norm $\|\cdot\|_{M}=\|\cdot\|_{L_{0}^{s^{\prime}}(\Omega)}$, where $s^{\prime}=s /(s-1)$, then the saddle-point weak formulation of problem (4) is:

find $(\mathbf{u}, p) \in \mathbf{X} \times M$ such that

$$
\begin{array}{ll}
a(\mathbf{u} ; \mathbf{u}, \mathbf{w})+b(\mathbf{w}, p)=(\mathbf{f}, \mathbf{w}) & \forall \mathbf{w} \in \mathbf{X} \\
b(\mathbf{u}, q)=0 & \forall q \in M
\end{array}
$$

where the bilinear form $b: \mathbf{X} \times M \rightarrow \mathbb{R}$ is defined by $b(\mathbf{u}, q)=-\int_{\Omega} q \operatorname{div} \mathbf{u} d \Omega$. Taking $\mathbf{w} \in \mathbf{V} \subset \mathbf{X}$ in (10a) it follows that the unique solution $\mathbf{u}$ of (10) exists. Existence and uniqueness of $p$, follows from the Babuska-Brezzi infsup condition (for the proof see Amrouche and Girault [2,3])

$$
\exists \beta>0: \inf _{q \in M} \sup _{\mathbf{w} \in \mathbf{X}} \frac{b(\mathbf{w}, q)}{\|q\|_{M}\|\mathbf{w}\|_{\mathbf{X}}} \geq \beta .
$$

Let $\mathcal{A}: \mathbf{X} \longrightarrow \mathbf{X}^{*}$ be the operator such that $\langle\mathcal{A}(\mathbf{u}), \mathbf{w}\rangle=a(\mathbf{u} ; \mathbf{u}, \mathbf{w})$. The following inequalities hold for all $\mathbf{u}, \mathbf{w} \in \mathbf{X}$ (see [4]): for $s \in(1,2)$,

$$
\begin{gathered}
\|\mathbf{u}-\mathbf{w}\|_{\mathbf{X}}^{2} \leq C_{s}\langle\mathcal{A}(\mathbf{u})-\mathcal{A}(\mathbf{w}), \mathbf{u}-\mathbf{w}\rangle\left(\|\mathbf{u}\|_{\mathbf{x}}+\|\mathbf{w}\|_{\mathbf{x}}\right)^{2-s} \\
\|\mathcal{A}(\mathbf{u})-\mathcal{A}(\mathbf{w})\|_{\mathbf{X}^{*}} \leq C_{s}\|\mathbf{u}-\mathbf{w}\|_{\mathbf{X}}^{s-1}
\end{gathered}
$$


while for $s \in[2, \infty)$,

$$
\begin{gathered}
\|\mathbf{u}-\mathbf{w}\|_{\mathbf{X}}^{s} \leq C_{s}\langle\mathcal{A}(\mathbf{u})-\mathcal{A}(\mathbf{w}), \mathbf{u}-\mathbf{w}\rangle \\
\|\mathcal{A}(\mathbf{u})-\mathcal{A}(\mathbf{w})\|_{\mathbf{X}^{*}} \leq C_{s}\|\mathbf{u}-\mathbf{w}\|_{\mathbf{X}}\left(\|\mathbf{u}\|_{\mathbf{X}}+\|\mathbf{w}\|_{\mathbf{X}}\right)^{s-2}
\end{gathered}
$$

where the constant $C_{s}$ is independent of $\mathbf{u}$ and $\mathbf{v}$. Moreover, the following $a$ priori estimate can be obtained (see [27]):

Lemma 1 There exist two positive constants $\overline{C_{i}}, i=1,2$ that depend on $s, \Omega$ and $\mathbf{f}$ such that, if $\mathbf{u}$ is a solution of (9), then $\|\mathbf{u}\|_{\mathbf{x}} \leq \overline{C_{1}}$. Suppose further that (11) holds, and let $(\mathbf{u}, p)$ be the solution of (10). Then $\|p\|_{M} \leq \overline{C_{2}}$.

Proof. Set $\mathbf{v}=\mathbf{u}$ in (9). Then $a(\mathbf{u} ; \mathbf{u}, \mathbf{u})=(\mathbf{f}, \mathbf{u})$. We have by (13) that $\|\mathbf{u}\|_{\mathbf{X}}^{s} \leq C_{s} a(\mathbf{u} ; \mathbf{u}, \mathbf{u})=C_{s}(\mathbf{f}, \mathbf{u}) \leq C_{s}\|\mathbf{f}\|_{\mathbf{X}^{*}}\|\mathbf{u}\|_{\mathbf{X}}$ which implies

$$
\|\mathbf{u}\|_{\mathbf{X}} \leq C_{s}^{\frac{1}{s-1}}\|\mathbf{f}\|_{\mathbf{X}^{*}}^{\frac{1}{s-1}}
$$

for $2<s \leq \infty$. Similarly for $1<s \leq 2$ we have by (12)

$$
\|\mathbf{u}\|_{\mathbf{X}}^{2} \leq C_{s} a(\mathbf{u} ; \mathbf{u}, \mathbf{u})\|\mathbf{u}\|_{\mathbf{X}}^{2-s}=C_{s}(\mathbf{f}, \mathbf{u})\|\mathbf{u}\|_{\mathbf{X}}^{2-s}
$$

which gives (14). The inf-sup condition (11) implies

$$
\|p\|_{M} \leq \frac{1}{\beta} \sup _{\mathbf{w} \in \mathbf{X}} \frac{b(\mathbf{w}, p)}{\|\mathbf{w}\|_{\mathbf{X}}}=\frac{1}{\beta} \sup _{\mathbf{w} \in \mathbf{X}} \frac{a(\mathbf{u} ; \mathbf{u}, \mathbf{w})-(\mathbf{f}, \mathbf{w})}{\|\mathbf{w}\|_{\mathbf{X}}} \leq \frac{1}{\beta}\left(\|\mathcal{A}(\mathbf{u})\|_{\mathbf{X}^{*}}+\|\mathbf{f}\|_{\mathbf{X}^{*}}\right) .
$$

The boundness of $\|p\|_{M}$ follows now from the boundness of $\|\mathbf{u}\|_{\mathbf{x}}$ which is a consequence of (12) and (13).

\section{Numerical Approximation}

Let $\mathbf{X}_{h}$ and $M_{h}$ be two finite dimensional spaces such that

$$
\mathbf{X}_{h} \subset \mathbf{X} \cap \mathbf{W}^{1, \infty}(\Omega) \text { and } M_{h} \subset M \cap L^{\infty}(\Omega) .
$$

We can approximate problem (10) as follows: find $\left(\mathbf{u}_{h}, p_{h}\right) \in \mathbf{X}_{h} \times M_{h}$ such that

$$
\begin{array}{ll}
a\left(\mathbf{u}_{h} ; \mathbf{u}_{h}, \mathbf{w}_{h}\right)+b\left(\mathbf{w}_{h}, p_{h}\right)=\left(\mathbf{f}, \mathbf{w}_{h}\right) & \forall \mathbf{w}_{h} \in \mathbf{X}_{h} \\
b\left(\mathbf{u}_{h}, q_{h}\right)=0 & \forall q_{h} \in M_{h}
\end{array}
$$

If a discrete inf-sup condition like (11) holds on the subspaces with a positive constant $\beta_{h}>0$ (possibly depending on $h$ ) then (16) has a unique solution 
which satisfies the error bound $[31,16]$

$$
\left\|\mathbf{u}-\mathbf{u}_{h}\right\|_{\mathbf{x}} \leq C\left(\beta_{h}^{-1}\right)\left\|\mathbf{u}-\mathbf{w}_{h}\right\|_{\mathbf{x}}, \quad \forall \mathbf{w}_{h} \in \mathbf{X}_{h}
$$

In the special case where Taylor-Hood finite elements of degree $k(\geq 2)$ for the velocity field and $k-1$ for the pressure were chosen (this couple satisfies the discrete inf-sup condition [44]), the following error estimate was proven in [6].

Theorem 2 Let $\mu$ obey the power-law models. Let $(\mathbf{u}, p) \in \mathbf{X} \times M$ be the unique solution of (10). Then we have:

(1) if $r \in(1,2]$ and, for all $h>0, \beta_{h} \geq \bar{\beta}>0$, then

$$
\begin{aligned}
& \left\|\mathbf{u}-\mathbf{u}_{h}\right\|_{\mathbf{W}^{1, r}(\Omega)} \leq C h^{\frac{k r}{2}}\left(\|\mathbf{u}\|_{\mathbf{W}^{k+1, r}(\Omega)}^{r / 2}+\|p\|_{W^{k, r^{\prime}}(\Omega)}\right) \\
& \left\|p-p_{h}\right\|_{L^{r^{\prime}}(\Omega)} \leq \hat{C}\left(\|\mathbf{u}\|_{\mathbf{W}^{k+1, r}(\Omega)},\|p\|_{W^{k, r^{\prime}}(\Omega)}\right) h^{\frac{k r(r-1)}{2}}
\end{aligned}
$$

where $C$ and $\hat{C}$ do not depend on $h$;

(2) if $r \in[2, \infty)$ and, for all $h>0, \beta_{h} \geq \bar{\beta}>0$, then

$$
\left\|\mathbf{u}-\mathbf{u}_{h}\right\|_{\mathbf{W}^{1, r}(\Omega)} \leq C\left(\|\mathbf{u}\|_{W^{1, \infty}(\Omega)},\|\mathbf{u}\|_{W^{k+1,2}(\Omega)},\|p\|_{W^{k, 2}(\Omega)}\right) h^{\frac{k}{r-1}} .
$$

In particular when $r=2$, the error is of the order of $h^{k}$, which conforms to the classical finite element approximation results [44]. Similar results in the case

of the Carreau-law model can be found in $[5,6]$. The discrete formulation(16) yields a system of non-linear algebraic equations by explicitly choosing the bases for $\mathbf{X}_{h}$ and $M_{h}$. The corresponding solution provides the velocity field that can be used for the tracking of the interfaces, as described in [33]. To treat the associated non-linear algebraic system we consider a Newton linearization strategy.

\subsection{Newton linearization}

For given $\mathbf{u}_{h} \in \mathbf{X}_{h}$, the following map defines a continuous functional on $\mathbf{X}_{h}$

$$
\mathbf{w}_{h} \longrightarrow\left\langle G\left[\mathbf{u}_{h}\right], \mathbf{w}_{h}\right\rangle=a\left(\mathbf{u}_{h} ; \mathbf{u}_{h}, \mathbf{w}_{h}\right)-\left(\mathbf{f}, \mathbf{w}_{h}\right), \quad \forall \mathbf{u}_{h} \in \mathbf{X}_{h}
$$

Then, the solution $\mathbf{u}_{h}$ of (16) satisfies

$$
\left\langle G\left[\mathbf{u}_{h}\right], \mathbf{w}_{h}\right\rangle+b\left(\mathbf{w}_{h}, p_{h}\right)-b\left(\mathbf{u}_{h}, q_{h}\right)=0, \quad \forall\left(\mathbf{w}_{h}, q_{h}\right) \in \mathbf{X}_{h} \times M_{h} .
$$

Let $I_{\mathbf{u}_{h}} G\left[\mathbf{v}_{h}\right]$ denote the Gateaux derivative of $G$ in the direction $\mathbf{v}_{h} \in \mathbf{X}_{h}$ evaluated at $\mathbf{u}_{h}$, that is

$$
\left\langle I_{\mathbf{u}_{h}} G\left[\mathbf{v}_{h}\right], \mathbf{w}_{h}\right\rangle=\lim _{\epsilon \rightarrow 0} \frac{1}{\epsilon}\left[a\left(\mathbf{u}_{h}+\epsilon \mathbf{v}_{h} ; \mathbf{u}_{h}+\epsilon \mathbf{v}_{h}, \mathbf{w}_{h}\right)-a\left(\mathbf{u}_{h} ; \mathbf{u}_{h}, \mathbf{w}_{h}\right)\right]
$$


Then the Newton method for solving (18) reads: for a given initial guess $\mathbf{u}_{h}^{(0)} \in \mathbf{X}_{h}$, for $k \geq 1$ until convergence, find a sequence $\left\{\left(\mathbf{u}_{h}^{(k)}, p_{h}^{(k)}\right)\right\} \subset \mathbf{X}_{h} \times M_{h}$ such that $\forall\left(\mathbf{w}_{h}, q_{h}\right) \in \mathbf{X}_{h} \times M_{h}$

$$
\left\langle I_{\mathbf{u}_{h}^{(k-1)}} G\left[\mathbf{u}_{h}^{(k)}-\mathbf{u}_{h}^{(k-1)}\right], \mathbf{w}_{h}\right\rangle+b\left(\mathbf{w}_{h}, p_{h}^{(k)}\right)-b\left(\mathbf{u}_{h}^{(k)}, q_{h}\right)=-\left\langle G\left[\mathbf{u}_{h}^{(k-1)}\right], \mathbf{w}_{h}\right\rangle .
$$

Note that it is not necessary to specify an initial guess $p^{(0)}$ for the pressure term. In the case of the power-law model:

$$
\left\langle I_{\mathbf{u}_{h}} G\left[\mathbf{v}_{h}\right], \mathbf{w}_{h}\right\rangle=a\left(\mathbf{u}_{h} ; \mathbf{v}_{h}, \mathbf{w}_{h}\right)+\left(\frac{r-2}{2}\right) \mu_{0}^{\frac{r-4}{2}}\left(\left[\nabla \mathbf{u}_{h} \cdot \nabla \mathbf{v}_{h}\right] \nabla \mathbf{u}_{h}, \mathbf{w}_{h}\right) .
$$

Similar calculations hold for the Carreau law. If we substitute (20) in (19), the Newton method reads:

given $\mathbf{u}_{h}^{(0)} \in \mathbf{X}_{h}$, for any $k \geq 1$ until convergence find $\left(\mathbf{u}_{h}^{(k)}, p_{h}^{(k)}\right) \in \mathbf{X}_{h} \times M_{h}$ such that:

$$
\begin{gathered}
a\left(\mathbf{u}_{h}^{(k-1)} ; \mathbf{u}_{h}^{(k)}, \mathbf{w}_{h}\right)+\left(\frac{r-2}{2}\right) \mu_{0}^{\frac{r-4}{2}}\left(\left[\nabla \mathbf{u}_{h}^{(k-1)} \cdot \nabla \mathbf{u}_{h}^{(k)}\right] \nabla \mathbf{u}_{h}^{(k-1)}, \mathbf{w}_{h}\right)-b\left(\mathbf{w}_{h}, p_{h}^{(k)}\right) \\
=\left(\frac{r-2}{2}\right) \mu_{0}^{\frac{r-4}{2}}\left(\left|\nabla \mathbf{u}_{h}^{(k-1)}\right|^{2} \nabla \mathbf{u}_{h}^{(k-1)}, \mathbf{w}_{h}\right)+\left(\mathbf{f}, \mathbf{w}_{h}\right)
\end{gathered}
$$$$
b\left(\mathbf{u}_{h}^{(k)}, q_{h}\right)=0 \quad \forall\left(\mathbf{w}_{h}, q_{h}\right) \in \mathbf{X}_{h} \times M_{h} .
$$

When implementing this iterative scheme, the initial guess of the Newton method is obtained after carrying out a few steps of the fixed point scheme that we describe below. Let us start from the solution $\mathbf{u}_{h, 0}^{(0)} \in \mathbf{X}_{h}$ of the linear Stokes problem; then we define the sequence $\mathbf{u}_{h, j}^{(0)} \in \mathbf{X}_{h}$ and $p_{h, j}^{(0)} \in M_{h}$ for $j \geq 1$ to be the solution of the following linearized discrete system:

$$
\begin{array}{lr}
a\left(\mathbf{u}_{h, j-1}^{(0)} ; \mathbf{u}_{h, j}^{(0)}, \mathbf{w}_{h}\right)+b\left(\mathbf{w}_{h}, p_{h, j}^{(0)}\right)=\left(\mathbf{f}, \mathbf{w}_{h}\right) & \forall \mathbf{w}_{h} \in \mathbf{X}_{h}, \\
b\left(\mathbf{u}_{h, j}^{(0)}, q_{h}\right)=0 & \forall q_{h} \in M_{h} .
\end{array}
$$

Then the initial guess for the Newton method is chosen $\mathbf{u}_{h}^{(0)}=\mathbf{u}_{h, j^{*}}^{(0)}$, where $j^{*}$ is the iteration index that satisfies a prescribed convergence criterion. Although it is only linearly convergent, this scheme provides a simple way to initialize the Newton method.

\section{Modeling of complex sedimentary basins}

In this section the effect of a non-Newtonian power law behaviour is tested on a single layer overburden during the diapir growing process. Furthermore we describe how to model some important phenomena that occur during basins 


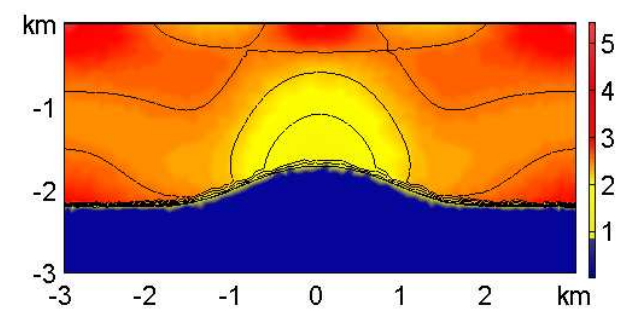

(a) $t=95 M a$

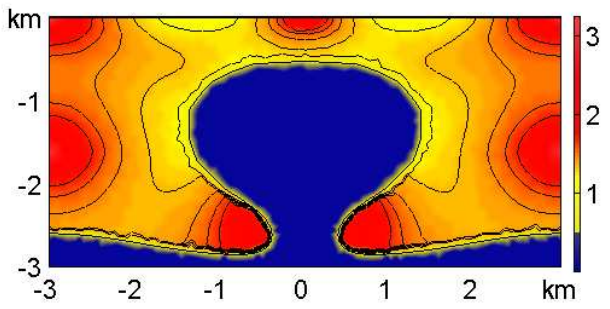

(c) $t=75 \mathrm{Ma}$

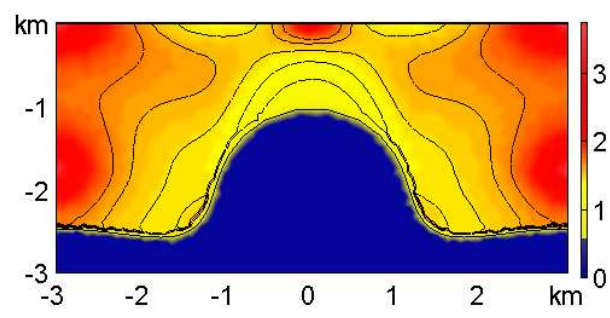

(b) $t=85 M a$

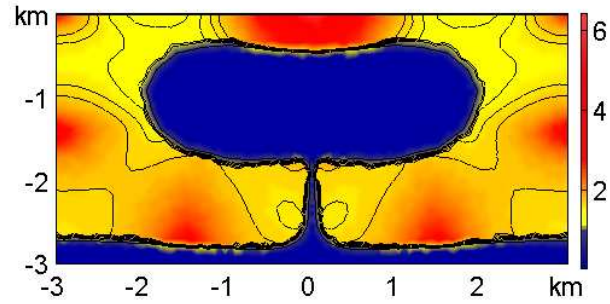

(d) $t=65 M a$

Figure 2. Evolution of a single salt diapir in a non-Newtonian overburden modeled with power-law rheology. The power law exponent is $r=1.7$. In these pictures the evolution of the apparent viscosity ( $\mathrm{Pas}$ ) (together with its isolines) of the overburden is shown.

geohistory, such as the kinematic of faults and lateral salt evacuation. We analyze here the effect of the power law rheology on the overburden behaviour. The gravitational instability of a buoyant viscous layer (salt) overlain by a $(r=1.7)$ non-Newtonian viscous layer is studied. As Figure 2 shows, the effect of the non-Newtonian rheology of the overburden is related to the apparent viscosity $\mu=\mu\left(|\mathbf{D}|^{2}\right)$. The diapir piercing the overburden makes the viscosity locally decrease changing the properties of surrounding material. A decrease in viscosity changes the tensional state of the overburden (due to the lower viscosity around the diapir), making the evolution of salt structures faster[17], and provides information on possible fault formation.

\subsection{Kinematic description of faults}

The description of the whole range of the rheological behaviours of rocks is very complex because it comprises a wide variety of deformation mechanisms. A macroscopic effect in the deformation of sedimentary basins is the kinematics induced by the presence of localized faulting. This is an important feature that has to be included in a comprehensive description of sedimentary basins. In this work we don't investigate the physical conditions leading to a fault 
generation, but we want to include the effects induced by the faults in the dynamics of a basin. Many sand-box experiments show a strict correlation between faults and diapirs growing in extensional basins. In order to be consistent with the fluid approach we model a fault as a shear band of lower viscosity with respect to the surrounding rocks. This approach, namely to locally change the material parameters in the zones where there is indication of some weakness, is commonly used in many fields in order to include effects that are not modeled by the basic framework. In this work we do not deal with the problem of finding an indicator for an arising weakness. This is also motivated by the very long time scales which characterize basin evolution. We assume that the weakness localization is given from different sources, such as geological studies which provide the location of faults and the time of their activation. Then we include this information in our simulation by placing a thin low viscosity shear band over the fault. In order to better understand the effect induced by a low viscosity shear band we consider the simple case shown in Figure 3(a), where the boundaries of the shear band are two straight lines of infinite length separating the shear band from the sediment. A Newtonian rheology is assumed and $\mu_{s b}$ indicates the viscosity. If the two sides of the faults are moving with velocities $\mathbf{V}_{1}$ and $\mathbf{V}_{2}$ respectively, both aligned with the tangent direction on the boundaries, a planar Couette flow is found inside the shear band. In this case we can compute the exact velocity inside the band. Let us denote by $n$ the distance from the left boundary measured along the perpendicular to the boundaries and by $L$ the distance between the two boundary lines. Then the exact solution for the velocity inside the shear band is

$$
\mathbf{V}(n)=\mathbf{V}_{1}+\mathbf{V}_{r} \frac{n}{L}
$$

where $\mathbf{V}_{r}=\mathbf{V}_{2}-\mathbf{V}_{1}$ is the relative slip velocity of one side of the fault with respect to the other. Because of the linear distribution of the velocity with respect to the position, the shear stress is constant inside the shear band and its value is $\tau=\frac{\mu_{s b}}{L} \mathbf{V}_{r}$. So the shear stress acting between the two sides of the fault is proportional to the relative slip velocity. The proportionality factor $\mu_{s b} / L$ can be tuned by choosing both $\mu_{s b}$ and $L$.

Figure 3 reports a computational example of extensional sedimentary basin: the shear band and also the computational mesh that follows the geometrical evolution of the system can be observed.

\subsection{Salt flux}

2D seismic surveys are deployed in large areas where 3D seismic is prohibitively expensive, and they are laid out perpendicular to the main geological structures. The interpretetion is done mostly in $2 \mathrm{D}$ because of the technological limitations of $2 \mathrm{D}$ visualization tools. This approximation loses accuracy when 


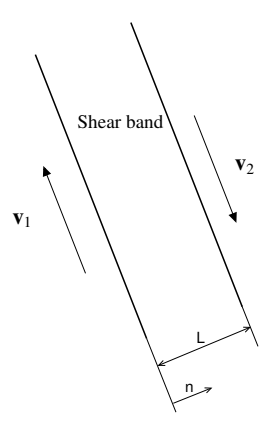

(a)

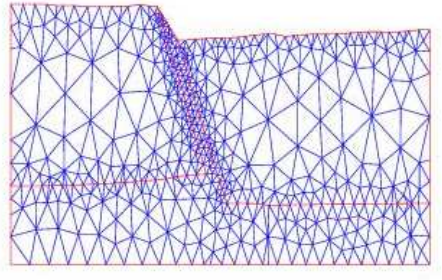

(b)

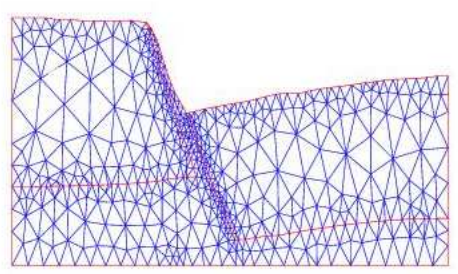

(c)

Figure 3. Development of a fault and computational mesh evolution.

evaporites are found inside the basins. This is caused by the high mobility of salt, as opposed to the higher strength of the overburden rocks.

Analyzing 3D-seismic data it can be noted that salt diapirs and tongues, are fully $3 \mathrm{D}$ structures and a reduction to a $2 \mathrm{D}$ section model can be a rough approximation in particular conditions. In order to take into account the full 3D migration of salt, geologists often reconstruct basins geohistory without respecting the constraint of area conservation in $2 \mathrm{D}$. This procedure can be numerically reproduced if the plain strain assumption is abandoned and a lateral salt flux (i.e. the movement of salt out of the section plane) is prescribed. From a computational point of view, this corresponds to a relaxation of the divergence constraint in two dimensions. In fact the velocity satisfies the incompressibility constraint in three dimension

$$
\frac{\partial u_{1}}{\partial x}+\frac{\partial u_{2}}{\partial y}+\frac{\partial u_{3}}{\partial z}=0
$$

If we assume that $\frac{\partial u_{3}}{\partial z}=S_{f}(\mathbf{x}, t)$ is a given function of space and time that prescribes lateral salt flux, we can rewrite (23) as div $\mathbf{u}=-S_{f}(\mathbf{x}, t)$, where $\mathbf{u}=$ $\left(u_{1}, u_{2}\right)^{T}$ is the unknown plane-velocity to be computed in a two-dimensional simulation. The reformulation of problem (4) that includes lateral salt flux reads: find $(\mathbf{u}, p)$ such that

$$
\begin{array}{ll}
-\operatorname{div}\left(\mu\left(\Pi_{D}(\mathbf{u})\right) \mathbf{D}(\mathbf{u})\right)+\nabla p=\mathbf{f} & \text { in } \Omega \\
\operatorname{div} \mathbf{u}=-S_{f}(\mathbf{x}, t) & \text { in } \Omega .
\end{array}
$$

We restrict our analysis to the following special class of flux function $S_{f}(\mathbf{x}, t)=$ $\sum_{i=1}^{N} S_{i}(\mathbf{x}) f_{i}(t)$, in which it is possible to decouple the spatial part from the temporal part and each addendum represents an event of salt evacuation. More precisely, $S_{i}(\mathbf{x})=\chi_{\Omega_{f_{i}}}$ is the characteristic function of a subdomain $\Omega_{f_{i}}$ (for instance the box in figure 4 ), $f_{i}(t)=g_{i} \chi_{\left[t_{i}^{s}, t_{i}^{e}\right]}$, for every $i=1, \ldots, N$ are characteristic functions of the time intervals $\left\{\left[t_{i}^{s}, t_{i}^{e}\right]\right\}_{i}$ of intensity $g_{i}$ that 


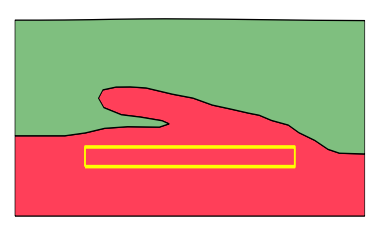

(a) $t=14 M a$

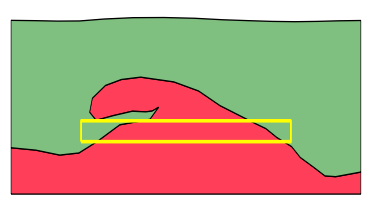

(b) $t=8 M a$

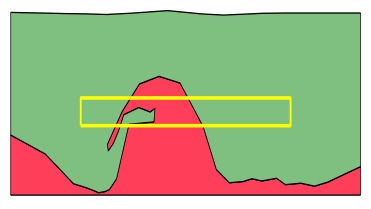

(c) $t=0 M a$

Figure 4. Evolution of a salt tongue in a extensional basin. The boxes represent the salt flux area. The evacuation intervals are $[18 M a, 14 M a],[9 M a, 8 M a],[4 M a$, $0 M a]$.

modulate the intensity in time of the salt flux, $N$ is the number of lateral flux events. Figure 4 shows a realistic case of a salt tongue in an extensional basin.

\section{Thermal modeling of a sedimentary basin}

Natural salt flow or halokinesis generally occurs at shallow crustal levels where temperature is relatively low, i.e. less than $423 \div 473 K$ (e.g., see [20]). Under these conditions the main deformation mechanism is creeping. Creep is the term given to the material deformation that occurs as a result of long term exposure to levels of stress that are below the yield or ultimate strength. Rather than failing suddenly with a fracture, the material permanently strains over a longer period of time until it finally fails. Creep does not happen upon sudden loading but the accumulation of creep strain in longer times causes failure of the material. Initially, the strain rate slows with increasing strain. This is known as primary creep. The strain rate eventually reaches a minimum and becomes near-constant. This is known as secondary or steady-state creep. In tertiary creep, the strain-rate exponentially increases with strain. The creep strain rate is typically the rate in the secondary stage. The link between stress and steady state creep rate depends on the creep mechanism. Commonly creeping deformation is governed by two mechanisms: dislocation creep and diffusional creep. At high stresses (relative to the shear modulus), creep is controlled by dislocation, while at lower stresses the diffusional creep is dominant. The rate of creep is well approximated by a power law dependence of the stress in the material $\mathbf{T}(\mathbf{u}, \theta)=\mu_{c} e^{-\frac{Q}{R \theta}}|\mathbf{D}|^{k} \mathbf{D}$, where $\mu_{c}$ is a parameter related to the material being crept and the sub-mechanism controlling creep, $Q$ is the activation energy for creep, $R$ the universal gas constant, $\theta$ the abso- 
lute temperature and $k$ a generic power-law exponent.

Numerous experiments have been conducted on a wide variety of natural salts to understand the creep behaviour of rocksalt. Investigated conditions cover temperatures in the range $293 \div 473 \mathrm{~K}$, strain rates down to $10^{10} \mathrm{~s}^{-1}$ and confining pressures typically up to $30 \mathrm{MPa}$. At experimental flow stresses below $\sim 15 \mathrm{MPa}$ and strain rates below $\sim 10^{-7} \mathrm{~s}^{-1}$ it is generally agreed that these experiments show flow by dislocation creep (see $[11,55]$ ).

\subsection{Physical Model}

Neglecting inertial forces, the equations of conservation of mass, momentum and thermal energy can be written in the Boussinesq approximation (see [26]) as

$$
\begin{aligned}
& -\operatorname{div} \mathbf{T}(\mathbf{u}, \theta)+\nabla p=\rho \mathbf{g}, \\
& \operatorname{div} \mathbf{u}=0 \\
& \frac{\partial \theta}{\partial t}+(\mathbf{u} \cdot \nabla) \theta=\operatorname{div}(\kappa \nabla \theta)+\frac{H}{\rho c_{p}}
\end{aligned}
$$

where $\theta$ is the absolute temperature, $\kappa$ the thermal diffusivity, $H$ the internal heating per unit of volume and $c_{p}$ the specific heat at constant pressure. To close the system we add the state equation for density

$$
\rho(\mathbf{x}, t)=\rho_{0}\left[1-\alpha\left(\theta(\mathbf{x}, t)-\theta_{0}\right)\right]
$$

where $\alpha$ is the thermal expansion coefficient and $\rho_{0}$ is the thermally unperturbed density at the reference temperature $\theta_{0}$, and the rheological model for viscosity

$$
\mu(\mathbf{x}, t)=\mu_{0} \exp \left(\frac{E+\rho g z V}{R \theta}-\frac{E_{0}+\rho_{0} g l_{0} V_{0}}{R \theta_{0}}\right),
$$

where $E$ is the activation energy, $V$ the activation volume, $z$ the depth, $g$ the value of gravity acceleration and $\mu_{*}$ is the thermally unperturbed viscosity, $E_{0}, l_{0}, V_{0}$ are reference values for activation energy, depth and activation volume. According to experimental data for the thermal regime, we assume as a value of $\Delta \theta$ the average geothermal gradient $(\partial \theta / \partial z)$ of $30 \mathrm{~K} / \mathrm{km}$. The thermal diffusivity of the salt is much higher (approximatively 5 times) than the diffusivity of the surrounding sediment, and this has a strong influence on the temperature distribution in and around the salt. For models of salt diapirism with a typical vertical length scale of $4 \mathrm{~km}$, the thermal Rayleigh number, $R a=\rho g \alpha \Delta \theta h^{3} / \kappa \mu$, is around unity, much smaller than the critical Rayleigh number (see [9]), and thermal buoyancy forces can be neglected. In addition, the thermal diffusion time $h^{2} / \kappa$, (where $h$ is the depth of the layer

and $\kappa$ is the thermal diffusivity) is much shorter than the turn-over time of 


\begin{tabular}{|c|c|c|c|}
\hline Symbol & Definition & Unit & Value \\
\hline$\kappa$ & thermal diffusivity & $\mathrm{m}^{2} \mathrm{~s}^{-1}$ & $5 \cdot 10^{-6}$ \\
\hline$\theta$ & temperature & $\mathrm{K}$ & $270 \div 800$ \\
\hline$\Delta \theta$ & temperature gradient & $\mathrm{K}$ & 30 \\
\hline$R$ & gas constant & $\mathrm{J} \mathrm{mol}^{-1} \mathrm{~K}^{-1}$ & 8.314 \\
\hline$\alpha$ & coeff. thermal expansion & $\mathrm{K}^{-1}$ & $10^{-5}$ \\
\hline$E$ & activation energy & $\mathrm{J} \mathrm{mol}^{-1}$ & $2 \cdot 10^{4}$ \\
\hline$V$ & activation volume & $\mathrm{m}^{3} \mathrm{~mol}^{-1}$ & $4 \cdot 10^{-6}$ \\
\hline$\rho_{0}$ & density & $\mathrm{kg} \mathrm{m}^{-3}$ & $2.2 \cdot 10^{3}$ \\
\hline$g$ & gravity acceleration & $\mathrm{ms}^{-2}$ & 9.8 \\
\hline$l_{0}$ & reference length & $\mathrm{m}$ & $5 \cdot 10^{3}$ \\
\hline$\mu_{0}$ & viscosity & $\mathrm{Pa} \cdot s$ & $10^{18}$ \\
\hline
\end{tabular}

Table 1

Characteristic values of parameters and measure units involved in thermal diapir evolution

the system. For example, taking $h=4 \mathrm{~km}$, and the accepted thermal diffusivity for salt $\left(\kappa=5 \cdot 10^{-6} \mathrm{~m}^{2} \mathrm{~s}^{-1}\right)$ the diffusion time scale is $10^{5} \mathrm{a}$, compared with $10-100 M a$ for a diapir to reach its mature stage [20]. This implies that thermal advection can be neglected and the temperature in and around the salt can be calculated from the steady-state heat diffusion equation. We neglect here possible effects of convection in ground water surrounding the salt. Following this physical assumption and considering the case in which the heat generation term $H$ is zero, equation (24c) reduces to

$$
\operatorname{div}(\kappa \nabla \theta)=0
$$

Thanks to the dimensional analysis, problem (24) reduces to (6) in which density and rheologic parameters are given by (25) and (26). Table 1 resumes the characteristic physical values for the thermal evolution of a sedimentary basin and involved in the dimensional analysis.

\subsection{The Mathematical Model}

We describe the salt deformation using a Stokes non-linear system in $\Omega(t) \subset$ $\mathbb{R}^{d}(d=2,3)$ with moving interface $\Gamma_{t}$ coupled with the thermal field. Also in this context the interface $\Gamma_{t}$ (see Figure 1 ), separating $\Omega^{+}$and $\Omega^{-}$is characterized as the loci of discontinuity of the density. Our model for diapiric growth can be formulated as the set of equations (24a),(24b), (27). They are com- 
pleted by the state equations (25) and (26) for $\rho$ and $\mu$ and by the advection equations for the thermally unperturbed density and viscosity

$$
\frac{\partial \rho_{0}}{\partial t}+\mathbf{u} \cdot \nabla \rho_{0}=0 \text { in } \Omega, \quad \frac{\partial \mu_{0}}{\partial t}+\mathbf{u} \cdot \nabla \mu_{0}=0 \text { in } \Omega
$$

and with the following initial and boundary conditions:

$$
\begin{aligned}
& \mathbf{u}=\mathbf{0}, \quad \nabla \theta \cdot \mathbf{n}=q \quad \text { on } \Gamma_{1}, \\
& \mathbf{u} \cdot \mathbf{n}_{2}=0, \quad(\mathbf{T}-p \mathbf{I}) \mathbf{n}_{2} \cdot \mathbf{t}_{2}=0, \quad \nabla \theta \cdot \mathbf{n}=0 \quad \text { on } \Gamma_{2}, \\
& (\mathbf{T}-p \mathbf{I}) \mathbf{n}_{3}=-p_{e} \mathbf{n}_{3}, \quad \theta=\theta_{\text {top }} \quad \text { on } \Gamma_{3}, \\
& \rho(\mathbf{x}, 0)=\rho_{0}, \quad \mu(\mathbf{x}, 0)=\mu_{0} \quad \text { in } \Omega_{0},
\end{aligned}
$$

where $\theta_{\text {top }}$ is the temperature at the free surface.

\subsection{Numerical scheme}

We propose a decoupled algorithm to solve numerically the system given by equations (24a), (24b), (27). In our fractional scheme, this system is split into two subsystems to be solved sequentially. To advance in time from $t^{n}$ to $t^{n+1}$ we first compute the thermal field:

$$
\begin{gathered}
\operatorname{div}\left(\kappa \nabla \theta^{n}\right)=0 \text { in } \Omega\left(t^{n}\right) \\
\nabla \theta^{n} \cdot \mathbf{n}=q \text { on } \Gamma_{1}, \quad \nabla \theta^{n} \cdot \mathbf{n}=0 \text { on } \Gamma_{2}, \quad \theta^{n}=\mathrm{T}_{e \text { top }} \text { on } \Gamma_{3}\left(t^{n}\right) .
\end{gathered}
$$

Then we solve the non-linear Stokes problem using the following fixed point iterations: for $k=1,2, \ldots$, until convergence

$$
\begin{array}{ll}
-\operatorname{div} \mathbf{T}\left(\mathbf{u}_{k}^{n}, \theta^{n}\right)+\nabla p_{k}^{n}=\rho\left(\theta^{n}\right) \mathbf{g} & \text { in } \Omega\left(t^{n}\right), \\
\operatorname{div} \mathbf{u}_{k}^{n}=0 & \text { in } \Omega\left(t^{n}\right), \\
\mu\left(\mathbf{x}, t^{n}\right)=\mu_{0} \exp \left(\frac{E+\rho g z V}{R \theta^{n}}-\frac{E_{0}+\rho_{0} g l_{0} V_{0}}{R \theta_{0}}\right) & \text { in } \Omega\left(t^{n}\right), \\
\rho\left(\mathbf{x}, t^{n}\right)=\rho_{0}\left[1-\alpha\left(\theta^{n}-\theta_{0}\right)\right] & \text { in } \Omega\left(t^{n}\right), \\
\mathbf{u}_{k}^{n}=\mathbf{0} & \text { on } \Gamma_{1}, \\
\mathbf{u}_{k}^{n} \cdot \mathbf{n}_{2}=0, \quad\left(\mathbf{T}-p_{k}^{n} \mathbf{I}\right) \mathbf{n}_{2} \cdot \mathbf{t}_{2}=0 & \text { on } \Gamma_{2}, \\
\left(\mathbf{T}-p_{k}^{n} \mathbf{I}\right) \mathbf{n}_{3}=-p_{e} \mathbf{n}_{3} & \text { on } \Gamma_{3}\left(t^{n}\right)
\end{array}
$$

Then, using a Lagrangian technique we track the interfaces in order to update the geometric structures (see [33]). Figure 5 shows the evolution of the tem- 


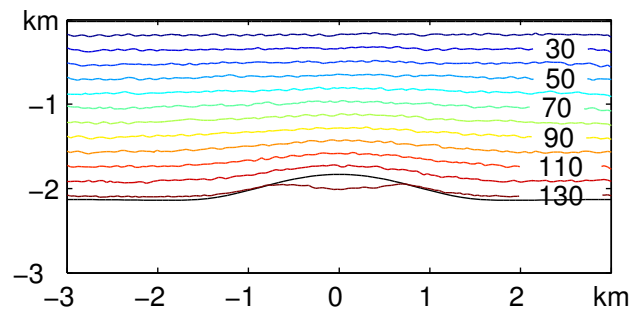

(a)

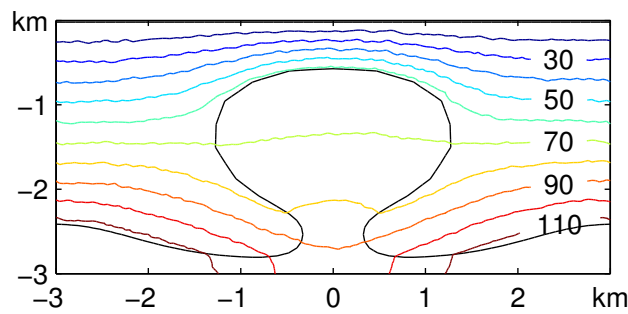

(c)

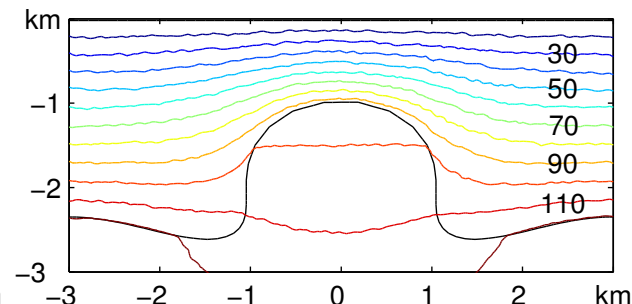

(b)

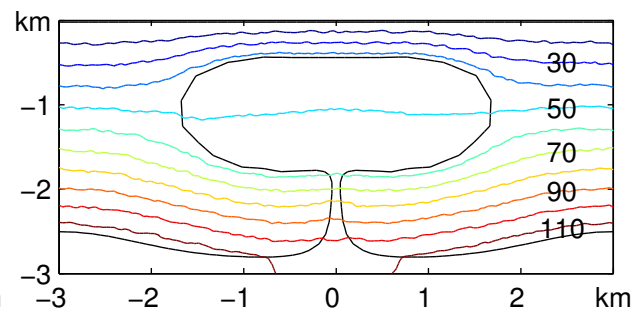

(d)

Figure 5. Thermal anomaly induced by a growing salt diapir in sedimentary basin. The figure represents the the isolines for temperature $\left({ }^{\circ} \mathrm{C}\right)$.

perature field in a sedimentary basin in presence of a growing diapir. Figure 6 shows the creeping strain rate in the salt diapir.

\section{$6 \quad$ Numerical results}

In this section we present the results of a simulation in which the proposed algorithms were applied to validate the restoration of a $2 \mathrm{~d}$ section taken in the Gulf of Mexico (see also [32]). This simulation is integrated in a more general scheme for the analysis of a sedimentary basin. The first step is to interpret the seismic data in order to build a reliable geometrical model of a present day configuration of the different layers in the basin. Then a retro-deformation is performed with the aid of the software tool 2DMove [29] and some intermediate configurations are produced under interpretative hypotheses formulated by the geologists. Finally, the proposed algorithms are applied to the initial configuration in order to validate the physical consistency of the geometrical reconstruction. 


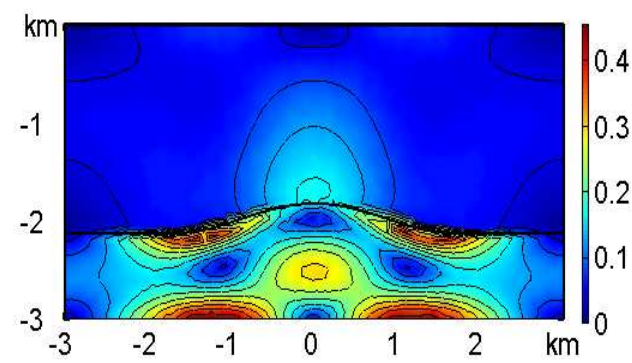

(a)

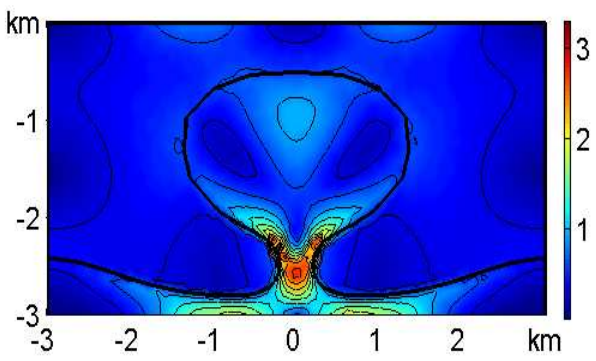

(c)

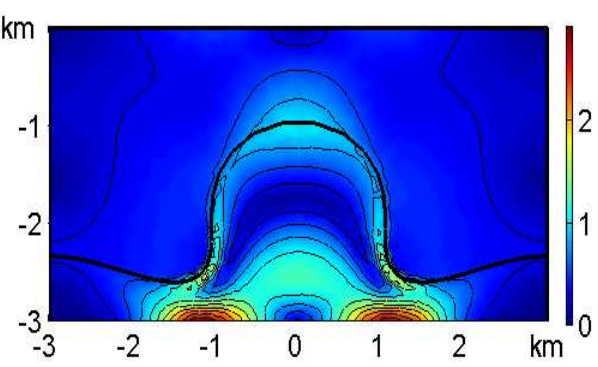

(b)

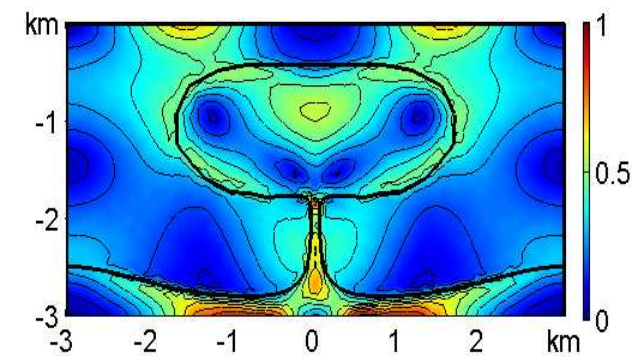

(d)

Figure 6. Creeping deformation of a salt diapir. Evolution of the strain rate $10^{-13}\left(s^{-1}\right)$.

\subsection{Geological Model and Restoration}

The area selected for the present study is located in the northeastern portion of the Gulf of Mexico basin. From Late Triassic to Jurassic the North American plate began separation from the South American and African plates. Separation was initiated by rifting, with the formation of grabens (see $[45,14]$ ). The basic structural setting of the basement in the Gulf of Mexico formed in Middle Jurassic time, with an important mantle upwelling and an extensional phase producing wide areas of both thin and thick crust (see [14]). The subsidence rate was higher for the thin crust than for the thick crust. Seawater is interpreted to have intermittently occupied the Gulf of Mexico. The high rate of evaporation of marine water caused the deposition of the salt found today in the Gulf of Mexico basin. It is believed that thick salt was deposited on the thin crust and in the structural lows of the thick crust (see [14]). Many geologists believe that oceanic crust formed in the center of the basin (see [45]) and the salt previously deposited in a single widespread basin, was split, by seafloor formation, into two regions: the northern region and the southern region (see [30]). Since Late Jurassic time, the basin was characterized by subsidence due to cooling and sediment loading (see [30]). During this period, the most important mechanisms for the deformation of the sedimentary cover was related to 


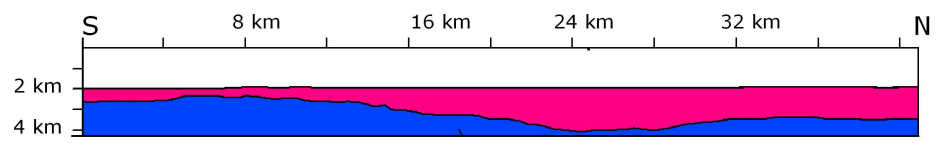

(a) Louann Salt

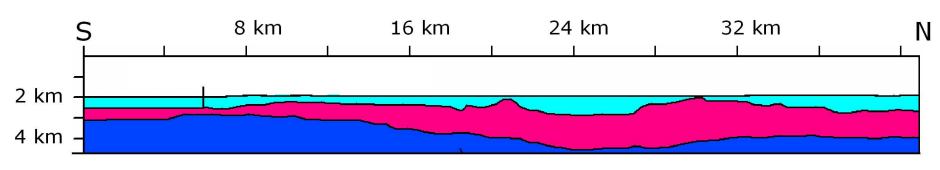

(b) Jurassic

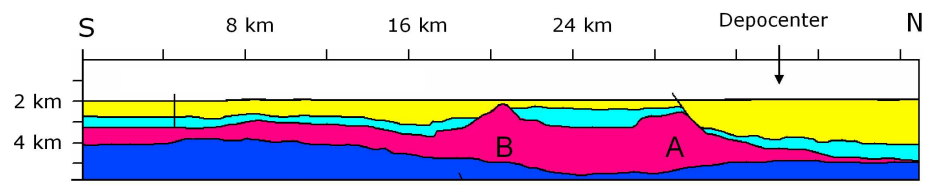

(c) Cretaceous phase 1

Figure 7. Kinematic reconstruction from Louann Salt deposition to Lower Cretaceous time features already active in the Mesozoic time

salt tectonics. From this point of view, the Gulf of Mexico basin presents some of the most numerous and complex salt structures in the world. In Jurassic time, sediment influx and progradation into the basin appears to have provided a differential loading that started the deformation of the autochthonous salt. The understanding of this early deformation of the Jurassic salt is one of the targets of the current study and an exceptionally clear example of the relationships between sediment progradation and related salt tectonics. In our reconstruction Upper Jurassic and Lower Cretaceous prograding wedges expelled most of the underlying salt. Starting in the Upper Jurassic, after the salt deposition (see Figure 7(a)), sediments covered the platform-slope system and salt started to flow (see Figure $7(\mathrm{~b})$ ). From Louann salt deposition time to Jurassic time the salt area was preserved. During Cretaceous, the sedimentary wedge experienced substantial growth: the progradation of sediments from the north caused a prevailing flow of salt southward. The salt movement toward the South was associated with depocenter migration in the same direction. During the Early Cretaceous (Creta phase 1, Figure 7(c)), sediments covered the area, especially in the northern depocenter. In this region, the area previously occupied by salt is now occupied by sediments. Salt flowed upward, forming diapirs and salt anticlines along the section and, from Jurassic to Cretaceous phase 1; the area occupied by salt remained the same. During Early 


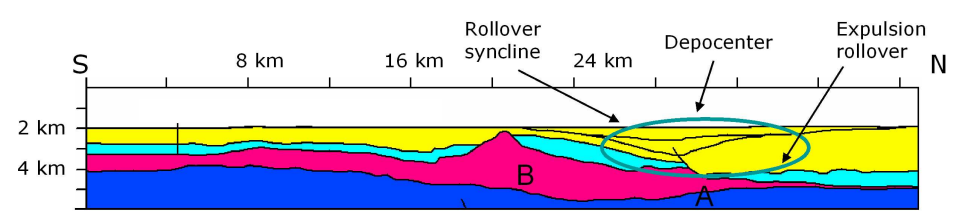

(a) Cretaceous phase 2

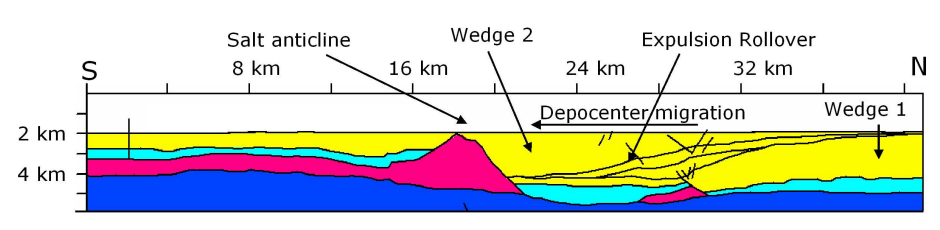

(b) Cretaceous phase 3

Figure 8. Kinematic reconstruction at the middle Cretaceous time (Cretaceous phase $3)$. Some of the salt is moved away from the $2 \mathrm{D}$ plane

Cretaceous time (Cretaceous phase 2, Figure 8(a)) new geometries developed with the rapid expulsion of salt from area A: the expulsion rollover and the rollover syncline. Depocenters migrated from the northern area towards the rollover syncline area. From Creta phase 1 time to Creta phase 2 time, the area of salt decreased because the area previously occupied by salt in zone A was now occupied by sediments in the rollover syncline.

From Early Cretaceous (Figure 7(c)) to middle Cretaceous (Cretaceous Phase 3, Figure 8) the total area of salt decreased considerably. The area previously occupied by salt was now occupied by sediments of wedge 2 . As the salt flowed towards the salt anticline, as well as out of the plane of the section, a new expulsion rollover developed (Figure 8). During the early Tortonian (Figure $9(\mathrm{~b})$ ), minor salt expulsion from salt anticlines towards the southern part of the basin occurred and caused activation of synsedimentary faults 1, 2 and 3 . During the Messinian (Figure 9(c)), the final salt expulsion occurred along the section causing the reactivation of the synsedimentary faults 1 and 2. From Messinian to the present-day (Figure 9(d)), salt did not move along section and the basin underwent regional subsidence. Our 2D kinematic reconstruction, described above, covers the evolution of the basin from the deposition of the salt to present-day. Synsedimentary structural deformation demonstrates strong relationships between sedimentation and salt kinematics as early as the deposition of the first post-salt sequences. For this reason, numerical forwardmodeling of salt dynamics, triggered by sediment loading, is the primary target of the model described in the following paragraphs. The aim is to dynamically reproduce the kinematics and the final geometries observed and described above. 


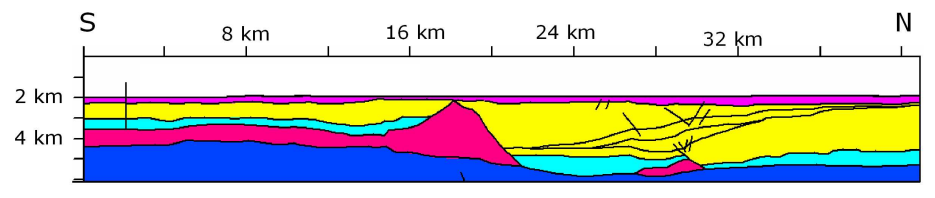

(a) late Oligocene

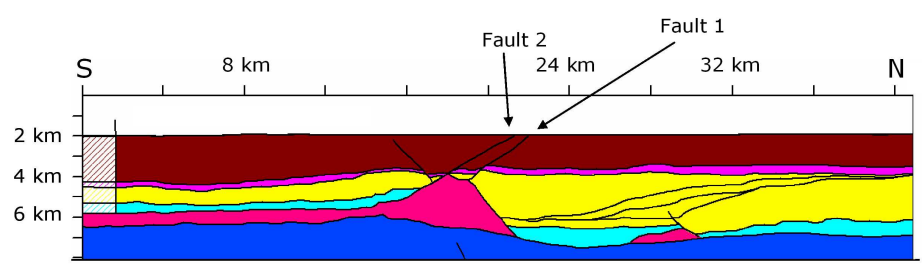

(b) early Tortonian

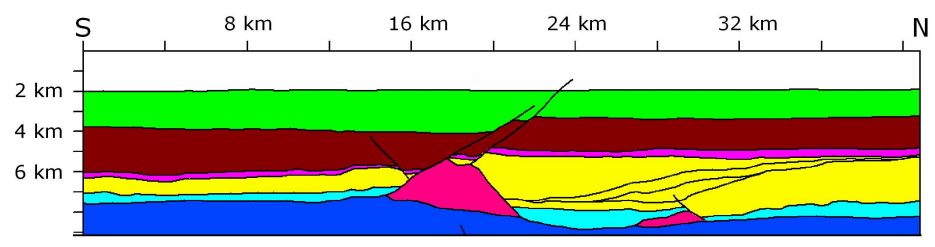

(c) Messinian

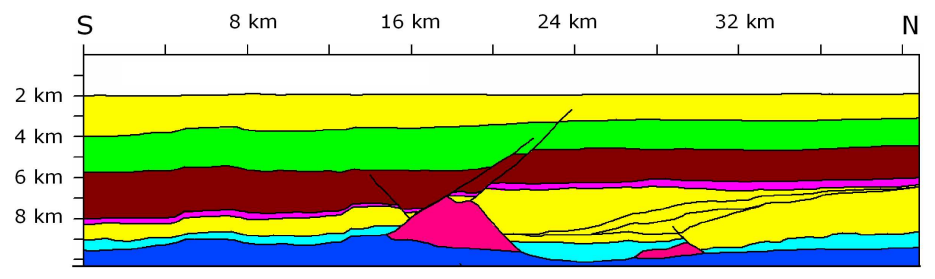

(d) Present Day

Figure 9. Kinematic reconstruction from Louann Salt deposition to Lower Cretaceous time features already active in the Mesozoic time

\subsubsection{Numerical Simulation}

The 2DMove key-frames associated to the main geological events, together with the geological interpretation, providing boundary conditions and rock properties, have been used as a guideline to reproduce the dynamic evolution. In detail, for each geological event, we performed some numerical tests to tune parameters that best reproduce the geometry coming from the restora- 
Figure 10. Example of computational mesh (4177 elements) at $6 \mathrm{Ma}$ (Messinian) in presence of faults system.

tion, at the end of the event. The salt was described in the simulation as a linear, viscous material with viscosity $\mu=10^{19}$ Pa.s and density $\rho=1900$ $K g / \mathrm{m}^{3}$. For all the other lithologies in the section, the densities were modeled as pseudo-plastic materials. The rheology parameters $\mu_{0}$ and $r$ depend on the rock composition and on depth to take into account the effects of the normal geothermal gradient. This dependency reflects a decrease of $\mu_{0}$ and $r$ with depth. Results of the numeric simulation are shown in Figure 11. In the initial geometry (Late Jurassic) the salt is already present in the section. Then the first two layers are gradually deposited between $160 \mathrm{Ma}$ and $132 \mathrm{Ma}$ and between $132 \mathrm{Ma}$ and $94 \mathrm{Ma}$ respectively. Under the load of those layers, the salt moves upwards where the lithostatic pressure is weaker and the resulting motion is in accord with models describing salt as a pressurized fluid ([47]). In the first phase, the sediments cover mainly the northern area initiating a salt anticline. In the second and third phases, we observe a rapid expulsion of salt concurrent with depocenter migration from the northern area toward the rollover syncline area, with wedges forming against the salt anticline. Moreover, during this stage the salt diapir is able to pierce the overburden. The next layer is deposited between $94 \mathrm{Ma}$ and $24 \mathrm{Ma}$ with no evident structural changes on the geological evolution of the section because it is thin and uniform, and does not introduce a significant change in the distribution of the lithostatic pressure. A significant deposition occurs between $24 \mathrm{Ma}$ and $11 \mathrm{Ma}$ inducing the development of the first important active fault emanating from the top of the salt. A similar effect is induced by the sedimentation that occurs between $11 \mathrm{Ma}$ and $6 \mathrm{Ma}$. The concurrent extension enhances the shear motion along the first fault and induces the activation of a second fault. The presence of the two faults makes the left part of the sedimentary layers move along the faults, thus squeezing the underlying salt out of the section until complete welding occurs. The entire region (basement included) subsides and the last layer uniformly covers the area. After the welding the faults become inactive and thus they are not propagated across the last depositional layer. Figure 10 shows some examples of the computational mesh with automatic refinement in presence of fault systems. 


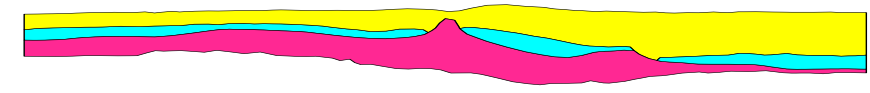

(a) Cretaceous (94 Ma)

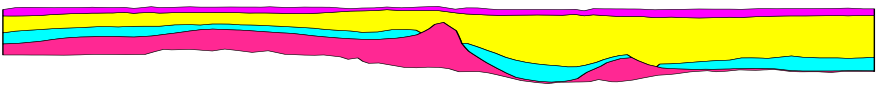

(b) Late Oligocene (24 Ma)

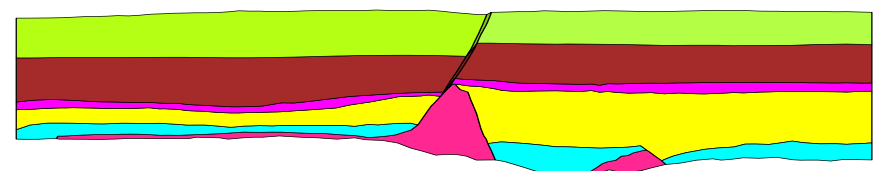

(c) Early Tortonian (11 Ma)

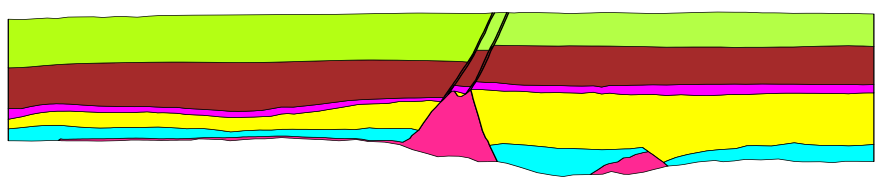

(d) Messinian (6 Ma)

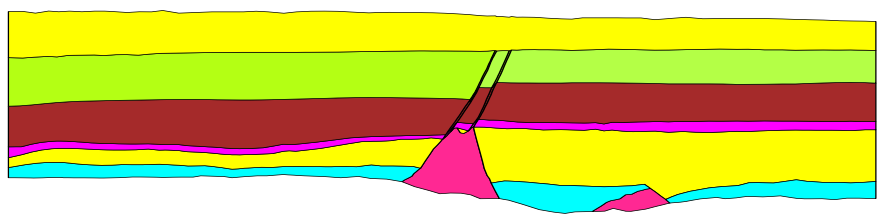

(e) Present Day

Figure 11. Dynamic evolution of sedimentary basin in Gulf of Mexico. For the dimensions we refer to the corresponding frames of the restoration (Figures 7, 8 and 9$)$. 


\section{Acknowledgments}

We acknowledge Drs P. Ruffo and D. Fragola from ENI E.\&P., Professors L. Formaggia and E. Miglio for their advises and collaboration on this project. This research has been supported by the "HPC on PSM" project in collaboration with ENI, E.\&P. This research has been made possible by the MIUR grant "Numerical Modelling for Scientific Computing and Advanced Applications".

\section{References}

[1] R.A. Adams. Sobolev Spaces. Academic Press, New York, 1975.

[2] C. Amrouche and V. Girault. Problèmes généralisés de Stokes. Portugal. Math., 49(4):463-503, 1992.

[3] C. Amrouche and V. Girault. Decomposition of vector spaces and application to the Stokes problem in arbitrary dimension. Czechoslovak Math. J., 44(119)(1):109-140, 1994.

[4] J. Baranger and K. Najib. Analyse numérique des écoulements quasi-newtoniens dont la viscosité obéit à la loi puissance ou la loi de carreau. Numer. Math., 58(1):35-49, 1990.

[5] J.W. Barrett and W.B. Liu. Finite element approximation of the $p$-Laplacian. Math. Comp., 61(204):523-537, 1993.

[6] J.W. Barrett and W.B. Liu. Finite element error analysis of a quasi-Newtonian flow obeying the Carreau or power law. Numer. Math., 64(4):433-453, 1993.

[7] J.W. Barrett and W.B. Liu. Quasi-norm error bounds for the finite element approximation of a non-Newtonian flow. Numer. Math., 68(4):437-456, 1994.

[8] M.A. Biot and H. Ode. Theory of gravity instability with variable overburden and compaction. Geophysics, 30 (2), 1965.

[9] F.H. Busse. Fundamentals of thermal convection. Mantle convection, plate tectonics and global dynamics. Gordon \& Breach, New York, 1989.

[10] P.J. Carreau. Rheological equations from molecular network theories. Trans. Soc. Rheol., 16:99-127, 1972.

[11] N.L. Carter and F.D. Hansen. Creep of rocksalt. Tectonophysics, 92:275-333, 1983.

[12] S. Chandrasekhar. Hydrodinamic and hydromagnetic instability. Oxford University Press, 1961.

[13] B. Daurè and S. Cloetingh. Numerical modelling of salt diapirism: influence of tectonic regime. Tectonophysics, 240, 1994. 
[14] L.M. Dobson and R.T. Buffler. Seismic stratigraphy and geologic history of jurassic rocks, northeastern gulf of mexico basin. AAPG Bull., 81(1):100-120, 1997.

[15] P. Fullsack. An arbitrary lagrangian eulerian formulation for creeping flows and its application in tectonic models. Geophys. J. Int., 120:1-23, 1995.

[16] V. Girault and P.-A. Raviart. Finite element methods for Navier-Stokes equations, volume 5 of Springer Series in Computational Mathematics. Springer-Verlag, Berlin, 1986.

[17] A.T. Ismail-Zadeh, H.E. Huppert, and J.R Lister. Analytical modelling of viscous diapirism through a strongly non-newtonian overburden subject to horizontal forces. J. Geodyn, 31:447-458, 2001.

[18] A.T. Ismail-Zadeh and C.J. Talbot. Dynamic restoration of profiles across diapiric salt structures: numerical approach and its application. Tectonophysics, $337: 129-151,2001$.

[19] M.P.A. Jackson, D.G. Roberts, and S. Snelson. Salt tectonics: a global perspective. AAPG Memoir, 65:454, 1995.

[20] M.P.A. Jackson and C.J. Talbot. External shapes, strain rates, and dynamics of salt structures. Geol.Soc.Amer.Bull., 97:305-323, 1986.

[21] P.K. Jensen. Calculations on thermal conditions around a salt diapir. Geophysical Prospecting, 31:481-489, 1983.

[22] P.K. Jensen. Analysis of the temperature field around salt diapirs. Geothermics, 19(3):273-283, 1990.

[23] S.H. Kirby and A.K. Kronenberg. Rheology of the lithosphere: selected topics. Rev. Geoph., 25:1219-1244, 1987.

[24] H.A. Koyi. Gravity, overturns, extension, and basement fault activation. Journal of Petroleum Geology, 12:117-242, 1991.

[25] H.A. Koyi. The shaping of salt diapirs. Journal of Structural Geology, 20:321$338,1998$.

[26] L.D. Landau and Lifshitz. Fluid Mechanics. Pergamon Press, 1993.

[27] L. Lefton and D. Wei. Penalty finite element approximations of the stationary power-law Stokes problem. J. Numer. Math., 11(4):301-322, 2003.

[28] I. Lerche. Temperature dependence of thermal conductivity and its impact on assessments of heat flux. Pageoph, 136:191-200, 1991.

[29] Midland Valley Ltd. 2dmove. http://www.mve.com.

[30] G. MacRae and S. Watkins. Basin architecture, salt tectonics and upper jurassic structural style, desoto canyon salt basin, northeastern gulf of mexico. AAPG Bull., 70(10):1809-1824, 1993. 
[31] M. Marion and R. Temam. Navier-Stokes equations: theory and approximation. In Handbook of Numerical Analysis, Vol. VI, Handb. Numer. Anal., VI, pages 503-688. North-Holland, Amsterdam, 1998.

[32] P. Massimi and G. Scrofani. 2D computational salt tectonics: Passive margins. Proceedings of the 25th Annual GCSSEPM Foundation, 2005.

[33] P. Massimi, G. Scrofani, and A. Quarteroni. An adaptive finite element method for modeling salt diapirism. M3AS, 16:587-614, 2006.

[34] P. Massimi, G. Scrofani, and F. Saleri. Multiphysics methods in basin modeling. In P. Diez and N. Wiberg, editors, Adaptive Modeling and Simulation, pages 281-290. CIMNE, 2005.

[35] U.T. Mello, R.N. Anderson, and G.D. Karner. Salt restrains maturation in subsalt plays. Oil and Gas Journal., 31:101-107, 1994.

[36] U.T. Mello, G.D. Karner, and R.N. Anderson. The role of salt rock in modifying the maturation history of sedimentary basins. Marine and Petroleum Geology, 12(7):697-716, 1995.

[37] B.M. Naimark and A.T. Ismail-Zadeh. Numerical approach to problems of gravitational instability of geostructures with advected material boundaries. Geophys. J. Int., 134, 1998.

[38] J.J. O'Brien and I. Lerche. The influence of salt domes on paleotemperature distributions. Geophysics, 49:2032-2043, 1984.

[39] R.G. Owens and T.N. Phillips. Computational Rheology. Imperial College Press, London, 2002.

[40] D. Peric and A.J.L. Crook. Computational strategies for predictive geology with reference to salt tectonics. Comp. Meth. Appl. Mech. Engng., 193:5195-5222, 2004 .

[41] Y. Podladchikov, C. Talbot, and A.N.B. Poliakov. Numerical-models of complex diapirs. Tectonophysics, 228(3-4):189-198, 1993.

[42] A.N.B. Poliakov and Y. Podladchikov. Diapirism and topography. Geophys. J. Int., 109, 1992.

[43] A.N.B. Poliakov, R. Vanbalen, Y. Podladchikov, B. Daudre, S. Cloetingh, and C. Talbot. Mechanics of active salt diapirism. Tectonophysics, 226(1-4):199-216, 1993.

[44] A. Quarteroni and A. Valli. Numerical approximation of partial differential equations, volume 23 of Springer Series in Computational Mathematics. Springer-Verlag, Berlin, 1994.

[45] A. Salvador. Late triassic-jurassic paleogeography and origin of gulf of mexico basin. AAPG Bull., 71(4):419-451, 1987. 
[46] D. Sandri. Sur l'approximation numérique des écoulements quasi-newtoniens dont la viscosité suit la loi puissance ou la loi de Carreau. RAIRO Modél. Math. Anal. Numér., 27(2):131-155, 1993.

[47] D.D. Schultz-Ela, M.P.A. Jackson, and B.C. Vendeville. Mechanics of active salt diapirism. Tectonophysics, 228(3-4):275-312, 1993.

[48] F. Sellig and G.C. Wallick. Temperature distribution in salt domes and surrounding sediments. Geophysics, 31(2):346-361, 1966.

[49] C.J. Talbot. Centrifuged models of gulf of mexico profiles. Marine and Petroleum Geology, 9:412-432, 1992.

[50] C.J. Talbot. Quo vadis tectonophysics? With a pinch of salt. Tectonophysics, $16: 1-20,1992$.

[51] C.J. Talbot and M.P.A. Jackson. Internal kinematics of salt diapirs. American Association of Petroleum Geologists Bulletin, 71(9):1068-1093, 1987.

[52] C.J. Talbot and M.P.A. Jackson. Salt tectonics. Scientific American, 255(8):7079, 1987.

[53] D. Waltham. Why does salt start to move? Tectonophysics, 282, 1997.

[54] John K. Warren. Evaporites. Springer-Verlag, 2005.

[55] W.R. Wawersik and D.H. Zeuch. Modeling and mechanistic interpretation of creep of rocksalt below 200 c. Tectonophysics, 121:125-152, 1986.

[56] R. Weijermars, M.P.A. Jackson, and B.C. Vendeville. Rheological and tectonic modeling of salt provinces. Tectonophysics, 217:143-174, 1993. 